\title{
Total Proctocolectomy
}

National Cancer Institute

\section{Source}

National Cancer Institute. Total Proctocolectomy. NCI Thesaurus. Code C103239.

Surgical removal of the entire colon, anus, and rectum. 